\title{
Design and Optimization of a Millimetre Wave Compact Folded Magic-T
}

\author{
Guang Hua, Jiefu Zhang, Jiudong Wu, and Wei Hong \\ State Key Laboratory of Millimetre Waves, School of Information Science and Engineering, Southeast University, \\ Nanjing 210096, China \\ Correspondence should be addressed to Guang Hua, huaguang@seu.edu.cn
}

Received 9 September 2011; Accepted 31 October 2011

Academic Editor: Zhongxiang Q. Shen

Copyright ( $\odot 2012$ Guang Hua et al. This is an open access article distributed under the Creative Commons Attribution License, which permits unrestricted use, distribution, and reproduction in any medium, provided the original work is properly cited.

A millimetre wave-folded magic-T junction compensated with metal cone is designed using a particle swarm optimization (PSO) algorithm. An off-centred metallic frustum was used to enhance the bandwidth and a metallic post is used to compensate the mismatched E-arm. The geometrical parameters of the frustum and the post are optimized by PSO. The optimized magic-T for W-band application is designed and tested. The design features are simple in structure and easy to fabricate. The $2 \%$ bandwidth with centre frequency of $94 \mathrm{GHz}$ and return loss less than $-20 \mathrm{~dB}$ is achieved.

\section{Introduction}

In communication and radar systems, waveguide junction components, such as couplers, magic-Ts, and orthogonal mode transformers (OMTs) have been used widely. Magic$\mathrm{T}$ features good isolation and power dividing performance, thus plays a key role in monopulse radar systems [1]. There have been various analyses of waveguide T-junctions using FDTD/matrix pencil method [2] and BCMM method [3]. FEM [4] and MoM [5] analysis of magic-T and folded magic-T compensated by offset metallic post of arbitrary height have also been reported. However, conventional methods require long computation time, and these magicTs demonstrate a narrow bandwidth at each port with return loss less than $-20 \mathrm{~dB}$. Recently, particle swarm optimization (PSO) was introduced in the optimization of electromagnetic problems [6]. Compared with conventional methods, PSO features fast convergence rate and the capability for multiple parameters. An X-band waveguide magic-T compensated by a stepping conducting cone was reported recently [7]. However, due to the reduction in size, it is very difficult to fabricate the stepping cone at $W$-band.

This paper presents the design and optimization of a millimetre folded waveguide magic- $\mathrm{T}$ junction compensated with an off-centred metallic frustum in the cavity region and a metallic post in the E-arm. A three-dimensional finite element method (FEM) is used to compute the scattering parameters of each port of the proposed magic- $\mathrm{T}$, and the calculated results were then sent to PSO code to evaluate the fitness. The PSO algorithm adjusts and updates the parameters accordingly until all parameters were optimized. The optimized folded magic- $\mathrm{T}$ junction was simulated and tested at $W$-band applications and demonstrated an over $2 \%$ $-20 \mathrm{~dB}$ reflection bandwidth. The design, optimization, and the measured performance of the proposed folded magic- $\mathrm{T}$ are described in the following.

\section{Magic-T Design}

The configuration of the proposed folded magic- $\mathrm{T}$ is shown in Figures 1 and 2. Ports 1 and 4 are E- and $\mathrm{H}$-arm waveguide ports-respectively, and ports 2 and 3 are two branch waveguide ports. The structure consists of four waveguide ports, a waveguide-folded $\mathrm{T}$ junction, a metallic frustum in the cavity region, and a metallic post in the Earm.

As magic- $\mathrm{T}$ junction itself is inherently mismatched; it requires compensation units. Here, a metallic frustum was employed to achieve complete impedance matching in the $\mathrm{H}$-arm and half-impedance matching in the E-arm, while 


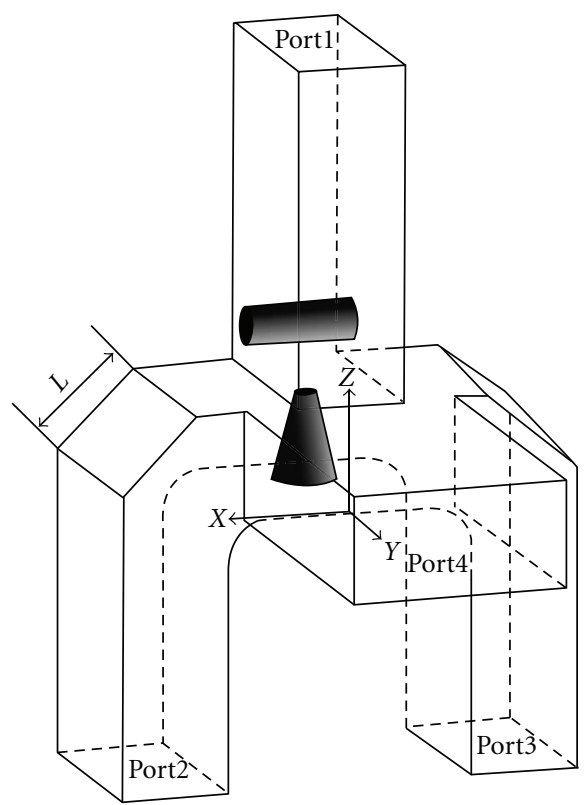

(a)

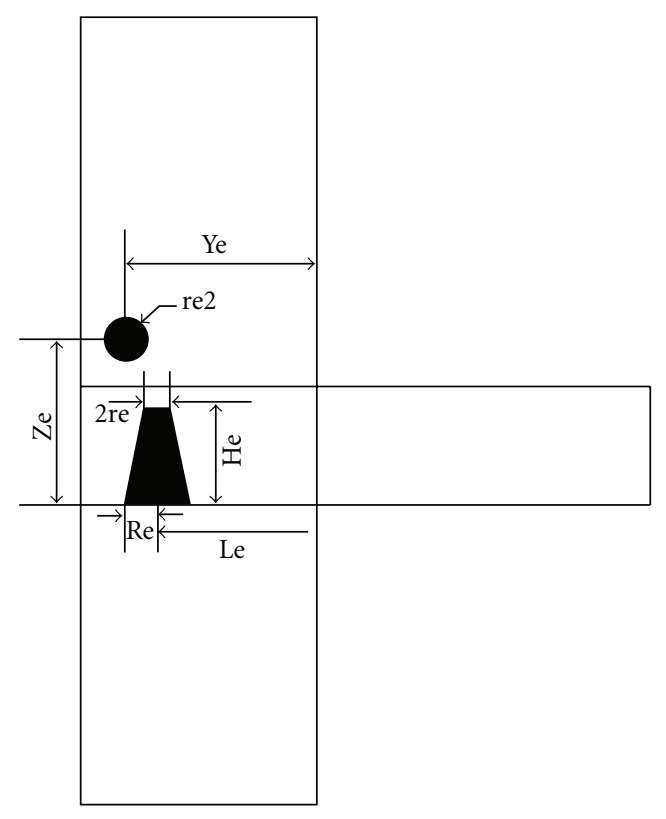

(b)

FIgURE 1: E-face-folded magic-T (a) geometry (b) parameters configuration.

a metallic post is employed to provide complete impedance matching of the E-arm. The frustum structure can effectively enhance the bandwidth performance of all the four ports. A detailed profile of the frustum and the post is shown in Figures 1(b) and 2(b). The optimization parameters of the Eand $\mathrm{H}$-plane-folded magic- $\mathrm{T}$ are the bottom and top radiuses of the frustum, the off-centre distance, the height, the radius, and position of the post.

As for E-plane-folded magic-T, the main waveguide is placed directly under the E-plane branch, and a 45 degree

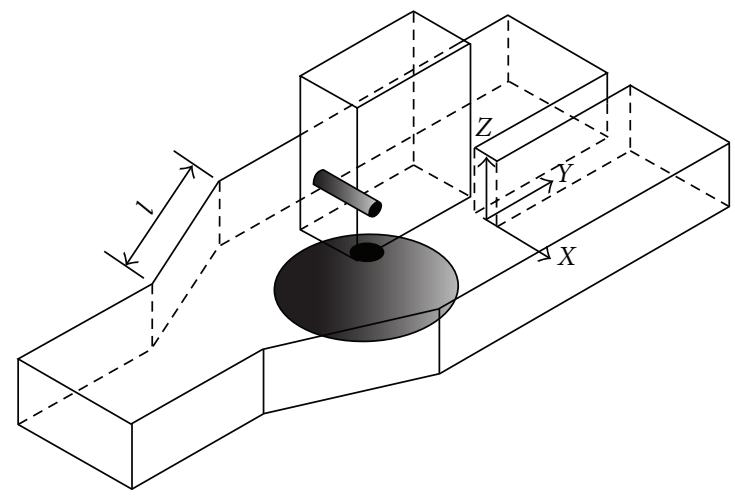

(a)

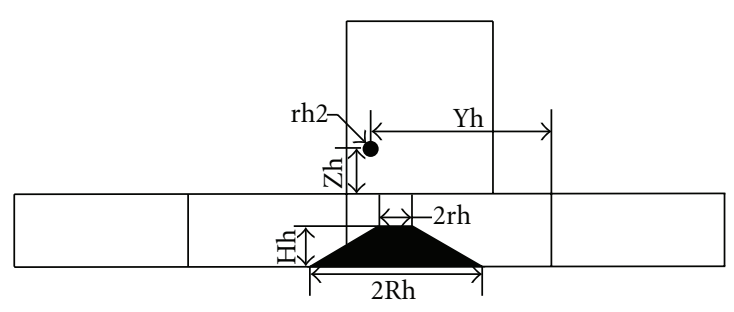

(b)

FIGURE 2: H-face-folded magic-T (a) geometry (b) parameters configuration.

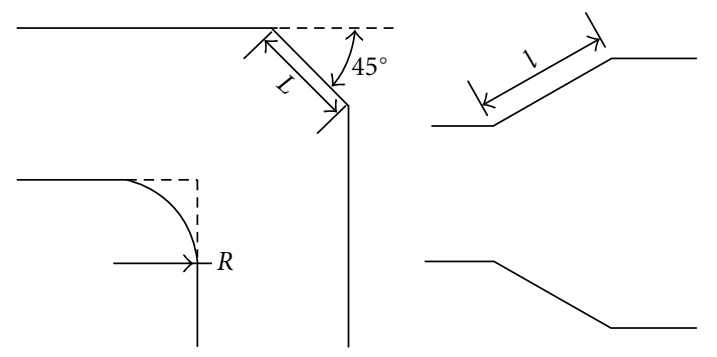

Figure 3: E-plane corner and H-plane taper configuration.

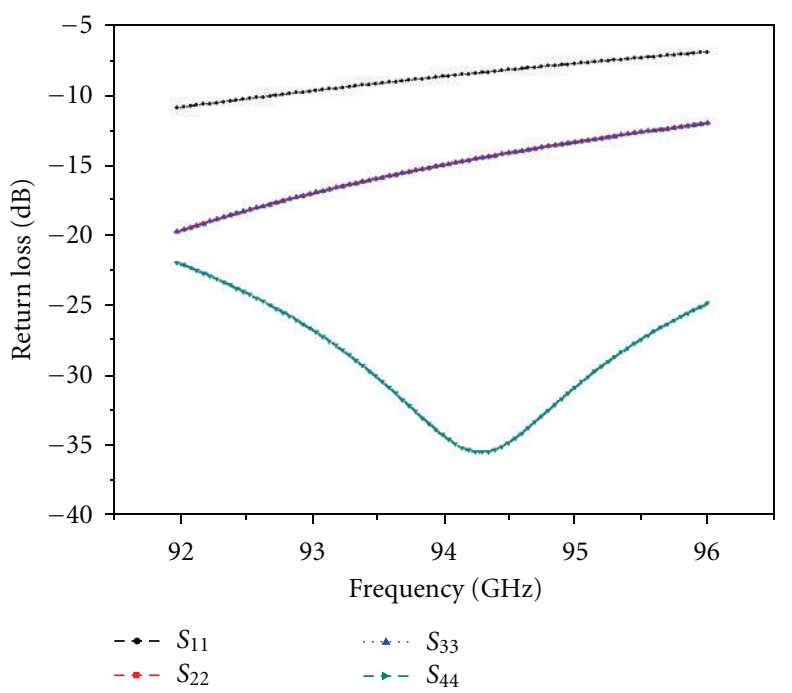

FIGURE 4: Half compensated E-FMT S-parameters of all ports. 


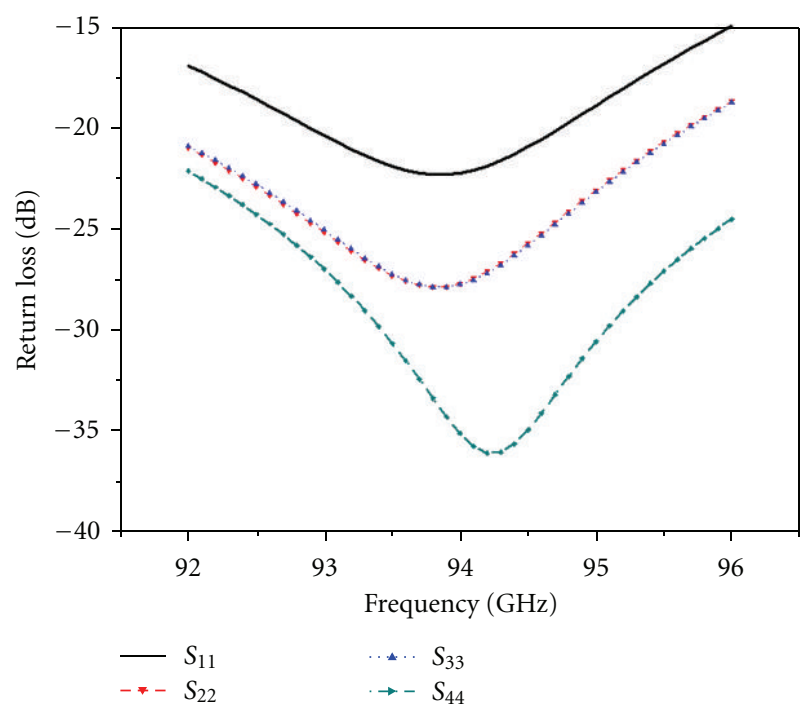

FIGURE 5: Fully compensated E-FMT refection coefficient of all ports.

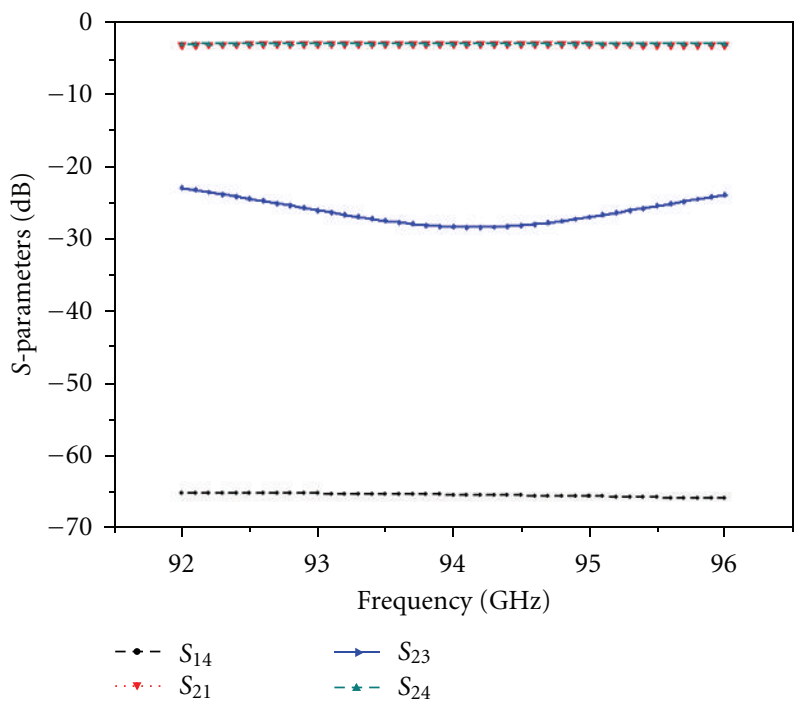

FIgURE 6: E-FMT isolation and transmission coefficients.

cutoff is used to match the impedance at the mitred corner, as shown in Figure 3. The length of the cutoff $L$ is $0.9 \mathrm{~b}$ [8], and the radius of inner corner is preoptimized as $0.5 \mathrm{~mm}$. The frustum is placed in the cavity region of the main waveguide to provide impedance matching to both $\mathrm{E}$ - and $\mathrm{H}$-plane branch. As for H-plane-folded magic- $\mathrm{T}$, a waveguide taper is employed to ensure enough space for compensation units in the cavity region; also, as shown in Figure 3, the length of the taper 1 is optimized with the frustum. In both E-plane and H-plane magic-T, the design consists of two steps: first, the $\mathrm{H}$-arm is fully compensated by the frustum and the E-arm is partly compensated; second, the E-arm is fully compensated by the off-centre metallic post. Capacitive posts reflect the electric field more effectively than inductive posts, but the latter is only half the length of the former and given the very

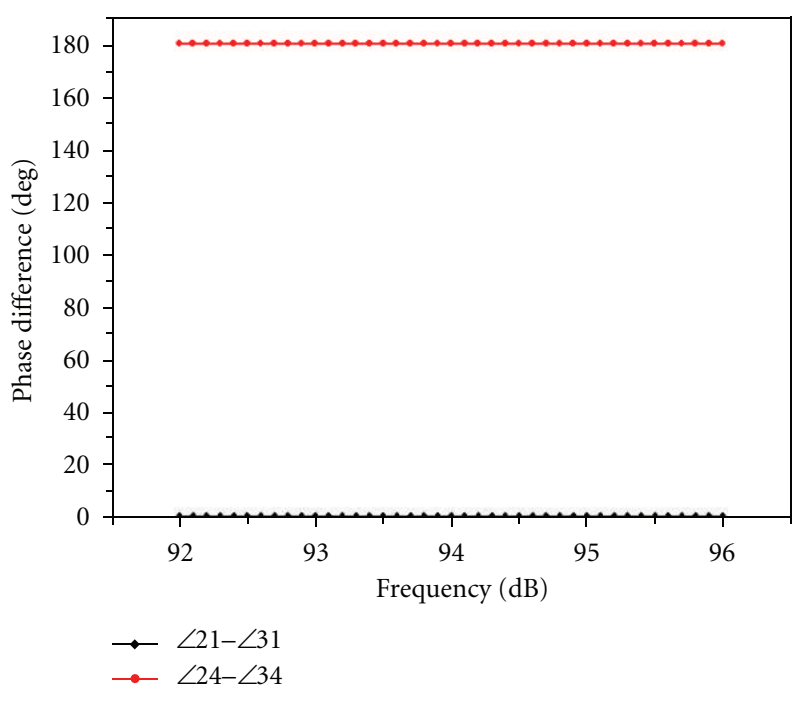

FIgURE 7: Phase characteristics of transmission coefficients.

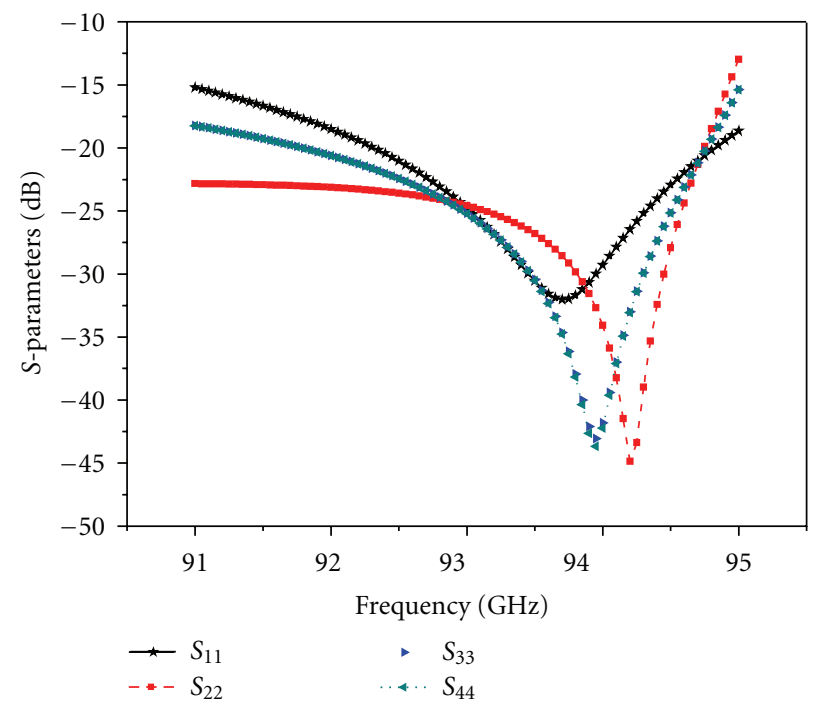

FIGURE 8: Fully compensated H-FMT S-parameters of all ports.

small radius, which is easier to manufacture and more stable in performance thus is used in both the two folded magic-Ts.

\section{Optimization}

$W$-band standard waveguides with the size of $a=2.54 \mathrm{~mm}$ and $b=1.27 \mathrm{~mm}$ were used to implement the magic-T. First, only the parameters of the frustum were optimized; as shown in Figure 1, there are four parameters: the radii of upper and lower section, the offset length, and the height. The modal is simulated by commercial software HFSS, and the results were fed into PSO MATLAB program with the optimization goal set to $S_{i i}<-20 \mathrm{~dB}$ within the frequency range of 93 to $95 \mathrm{GHz}$. VB (Microsoft Visual Basic) script is employed to connect the MATLAB and HFSS simulations in such an iteration that the initial model parameters in the 


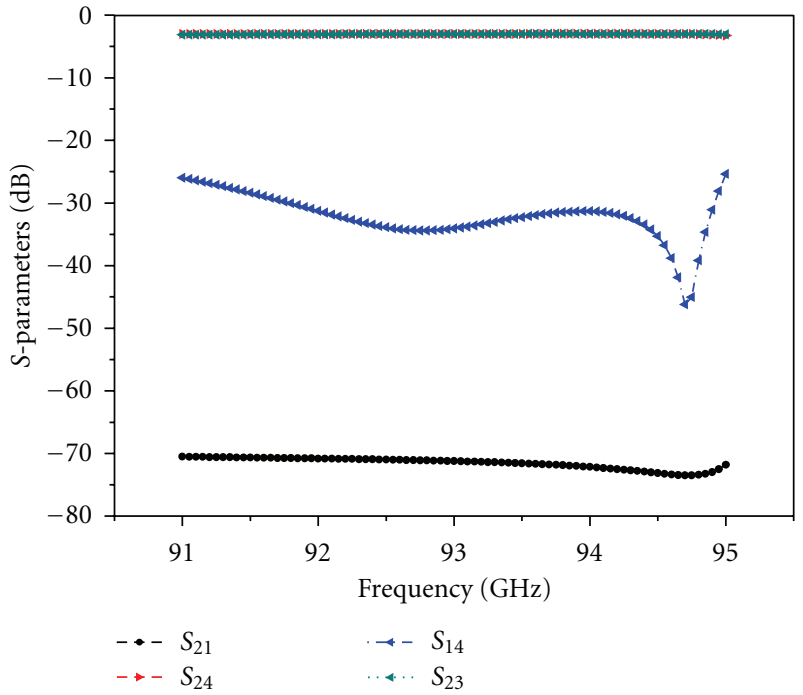

FIGURE 9: H-FMT isolation and transmission coefficients.

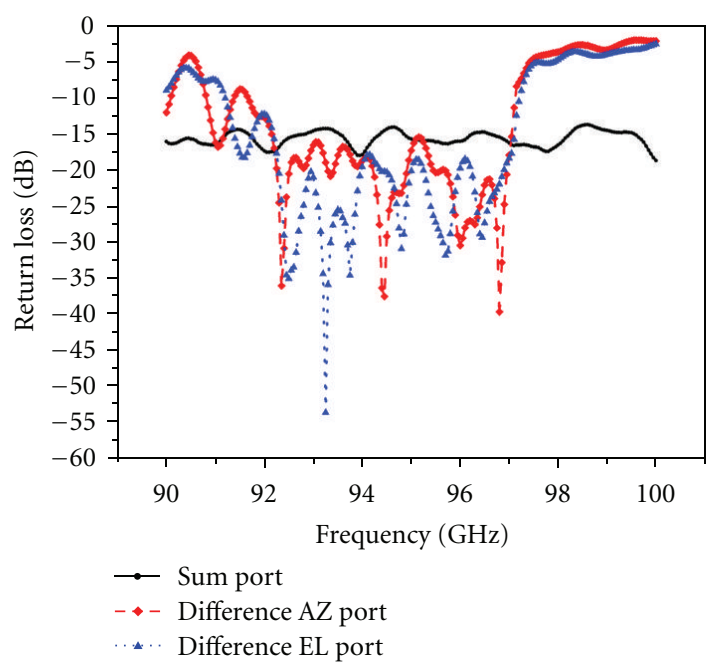

Figure 10: Measured return loss of the sum-difference device with horn.

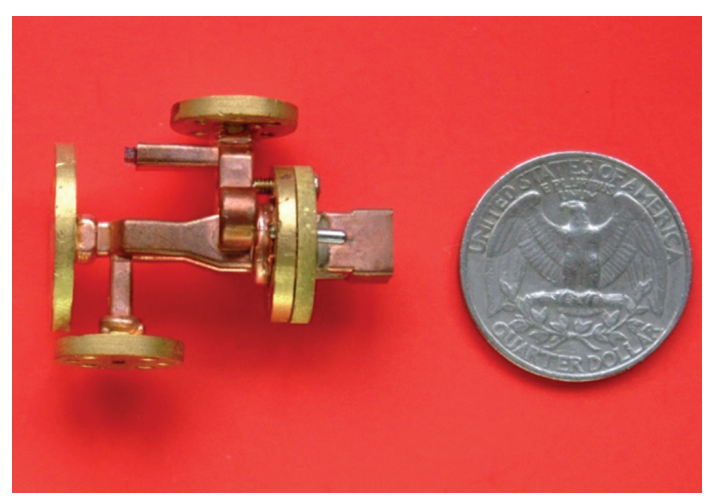

FIgURE 11: Photo of sum-difference device with horn.

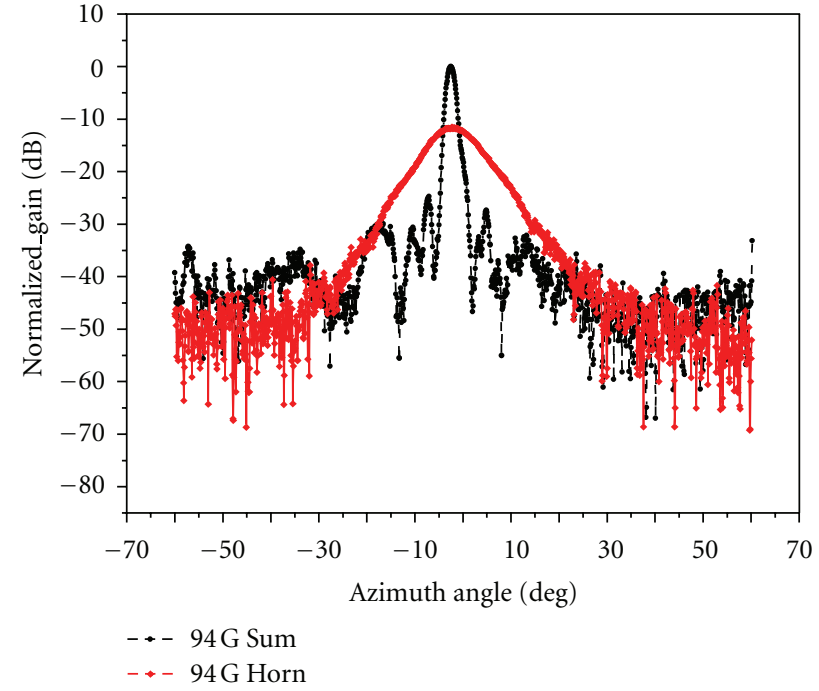

Figure 12: Antenna gain of sum signal and standard horn at $94 \mathrm{GHz}$.

script are simulated in HFSS to get an $S$-matrix, which is sent to the MATLAB to evaluate the fitness function and update the model parameters, and finally the updated model parameters are fed back into HFSS to get a renewed $S$-matrix. This iteration will carry on until the $S$-matrix values meet the set goals.

According to PSO algorithm, the parameters were updated using the following rule:

$$
\begin{aligned}
v_{i d}(t+1)= & w \times v_{i d}(t)+c_{1} \times \operatorname{rand}() \times\left(p_{i d}(t)-x_{i d}(t)\right) \\
& +c_{2} \times \operatorname{rand}() \times\left(p_{g d}(t)-x_{i d}(t)\right), \\
x_{i d}(t+1)= & x_{i d}(t)+v_{i d}(t+1), \quad 1 \leq i \leq N, 1 \leq d \leq M .
\end{aligned}
$$

The constants $c_{1}$ and $c_{2}$ are 1.494, and the weighting term $w$ decreases from 0.729 to 0.6 with 50 iterations. After the $\mathrm{H}$ plane waveguide branch was optimized, the post was added to the E-arm without entering the cavity region of the main waveguide. The post radius and offset coordination in $y$-and $z$-axes were optimized using the same equation. The total number of parameters optimized was seven. As for H-planefolded magic- $\mathrm{T}$, the taper length was also optimized in the first step; there was one more parameter.

\section{Results}

The optimized parameters were obtained after 87 iterations for E-plane-folded magic- $\mathrm{T}$ and 102 iterations for H-planefolded magic-T. The total simulation time on an Intel Core 2 2.1 GHz computer is 12.5 hours (frequency sweep step set to $0.1 \mathrm{GHz}$ ). However, a gradient method of the Quasi Newton optimizer is adopted in the HFSS; it suffers from the possible presence of local minima and numerical noise. It is nothing but $S_{44}>-10 \mathrm{~dB}$ with 44.25 computing hours. Table 1 shows the final results of all parameters after the PSO process. 


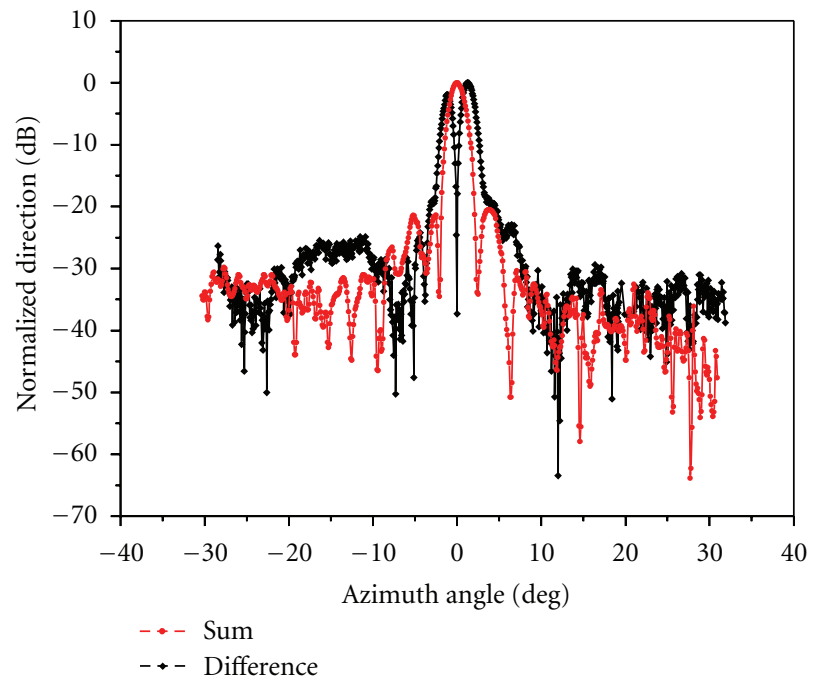

Figure 13: Normalized Direction of sum-difference signal at $94 \mathrm{GHz}$.

TABLE 1: Optimized value of all parameters.

\begin{tabular}{lcc}
\hline Parameter & Range $(\mathrm{mm})$ & Optimized $(\mathrm{mm})$ \\
\hline Re & {$[0.3,1.3]$} & 0.36 \\
re & {$[0.1,0.3]$} & 0.14 \\
Le & {$[-2,2]$} & 1.73 \\
$\mathrm{He}$ & {$[0.2,1.2]$} & 1.04 \\
re2 & {$[0.1,0.4]$} & 0.23 \\
Ye & {$[-2.4,-0.1]$} & -2.06 \\
Ze & {$[1.3,3.3]$} & 1.78 \\
l & {$[2,6]$} & 3.02 \\
Rh & {$[0.5,2]$} & 1.50 \\
rh & {$[0.1,0.3]$} & 0.28 \\
Lh & {$[0.5,6.5]$} & 2.70 \\
Hh & {$[0.2,1.2]$} & 0.70 \\
rh2 & {$[0.1,0.4]$} & 0.11 \\
Yh & {$[1.2,4]$} & 1.41 \\
Zh & {$[1.3,4.3]$} & 2.01 \\
\hline
\end{tabular}

Figure 4 shows the simulated reflection coefficients of the half-compensated E-plane-folded magic- $\mathrm{T}$ (only frustum and without post). It shows that the frustum alone has fully matched port 3 (H-arm) in the frequency range of $92 \mathrm{GHz} \sim$ $96 \mathrm{GHz}$, and the E-arm is only half-compensated. Figure 5 shows the reflection coefficients after the post is added. The reflection coefficients of all ports are below $-20 \mathrm{~dB}$ with $2.7 \%$ bandwidth. Figures 6 and 7 show the transmission and isolation coefficients, as well as the phase characteristics of EFMT. The power from $\mathrm{E}$ - or $\mathrm{H}$-arms is divided equally into two ports of the main waveguide, and the phase difference is, respectively, 0 and 180 degrees. Similarly, Figures 8, 9, and 10 show the reflection, isolation, and transmission coefficients, as well as the measured return loss of the fully compensated sum-difference device with horn. The bandwidth with $S_{11}<$ $-20 \mathrm{~dB}$ of the H-FMT is $2.1 \%$ with the centre frequency of $94 \mathrm{GHz}$. The magnitude differences of the transmission coefficients of the two main waveguide ports within $0.1 \mathrm{~dB}$, and measured ports return losses of the compensated sumdifference device with horn are less $-15 \mathrm{~dB}$, respectively.

An inverse Cassegrain antenna at millimeter waveband with the sum-difference network comprised of the proposed folded magic- $\mathrm{T}$ were also fabricated and tested. Figure 11 shows the photo of sum-difference device with horn. Figures 12 and 13 show the tested sum and difference beam, compared to the gain of a standard horn antenna. The antenna shows good gain and beamwidth characters in the frequency band of 93 to $95 \mathrm{GHz}$, as well as an acceptable null depth $(<-30 \mathrm{~dB})$. However, due to the limitations of the fabrication precision, frequency shift is observed.

\section{Conclusions}

A HFSS (Ansoft) and PSO (Matlab) cooperating simulation was utilized to optimize waveguide magic-T junctions. The simulation time has been reduced effectively due to the fast convergence feature of PSO. A novel $W$-band-folded magic- $\mathrm{T}$ compensated by a frustum and a metallic post was designed. The compensational units were simple in structure and easy to fabricate, and the operation bandwidth reaches more than $2.0 \%$ with the center frequency of $94 \mathrm{GHz}$. Compared with other $94 \mathrm{GHz}$ magic- $\mathrm{T}$ for monopulse radar applications, this design effectively enhanced the bandwidth and still maintains good performance $\left(S_{i i}<-20 \mathrm{~dB}\right)$ among all ports. The inversed Cassegrain antenna with sum and difference network comprised of the proposed folded magic$\mathrm{T}$ has been tested and has shown narrow beamwidth, high gain, and acceptable null depth of the difference beam, thus proved the proposed design to be feasible in millimeter monopulse applications.

\section{References}

[1] J. Uher, J. Bornemann, and U. Rosenberg, Waveguide Components for Antenna Feed Systems: Theory and CAD, Artech House, Norwood, Mass, USA, 1993.

[2] J. Ritter and F. Arndt, "Efficient FDTD/Matrix-pencil method for the full-wave scattering parameter analysis of waveguiding structures," IEEE Transactions on Microwave Theory and Techniques, vol. 44, no. 12, pp. 2450-2456, 1996.

[3] J. M. Reiter and F. Arndt, "Rigorous analysis of arbitrarily shaped $\mathrm{H}$ - and E-plane discontinuities in rectangular waveguides by a full-wave boundary contour mode-matching method," IEEE Transactions on Microwave Theory and Techniques, vol. 43, no. 4, pp. 796-801, 1995.

[4] Z. Shen, C. L. Law, and C. Qian, "Hybrid finite-elementmodal-expansion method for matched magic T-junction," IEEE Transactions on Magnetics, vol. 38, no. 2, pp. 385-388, 2002.

[5] A. A. San Blas, F. Mira, V. E. Boria, B. Gimeno, M. Bressan, and P. Arcioni, "On the fast and rigorous analysis of compensated waveguide junctions using off-centered partial-height metallic posts," IEEE Transactions on Microwave Theory and Techniques, vol. 55, no. 1, pp. 168-174, 2007.

[6] J. Robinson and Y. Rahmat-Samii, "Particle swarm optimization in electromagnetics," IEEE Transactions on Antennas and Propagation, vol. 52, no. 2, pp. 397-407, 2004. 
[7] K. C. Hwang, "Design and optimization of a broadband waveguide magic-T using a stepped conducting cone," IEEE Microwave and Wireless Components Letters, vol. 19, no. 9, Article ID 5200440, pp. 539-541, 2009.

[8] G. L. Ragan, Microwave Transmission Circuits, vol. 9 of MIT Radiation Laboratory Series, McGraw-Hill, New York, NY, USA, 1948. 

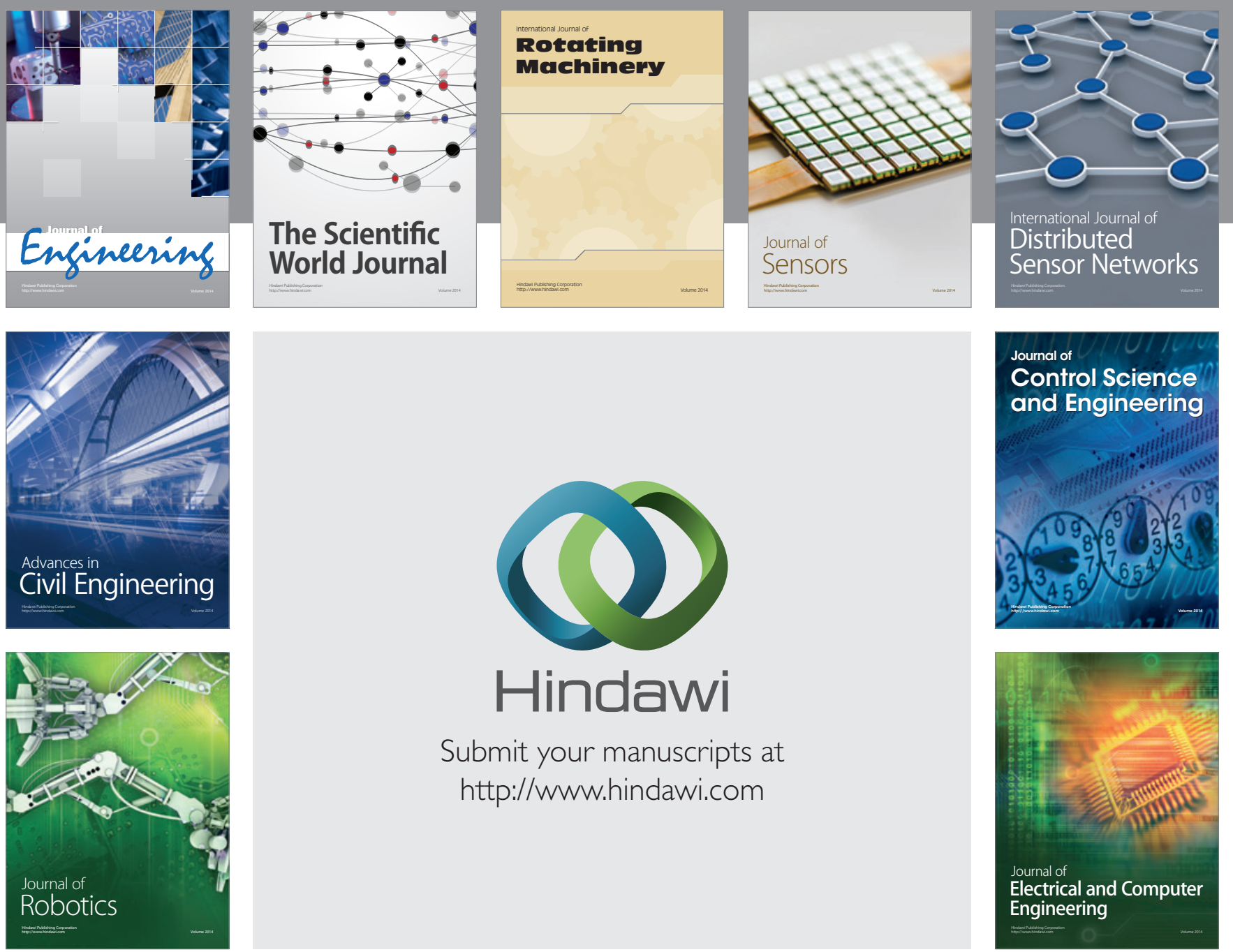

Submit your manuscripts at

http://www.hindawi.com
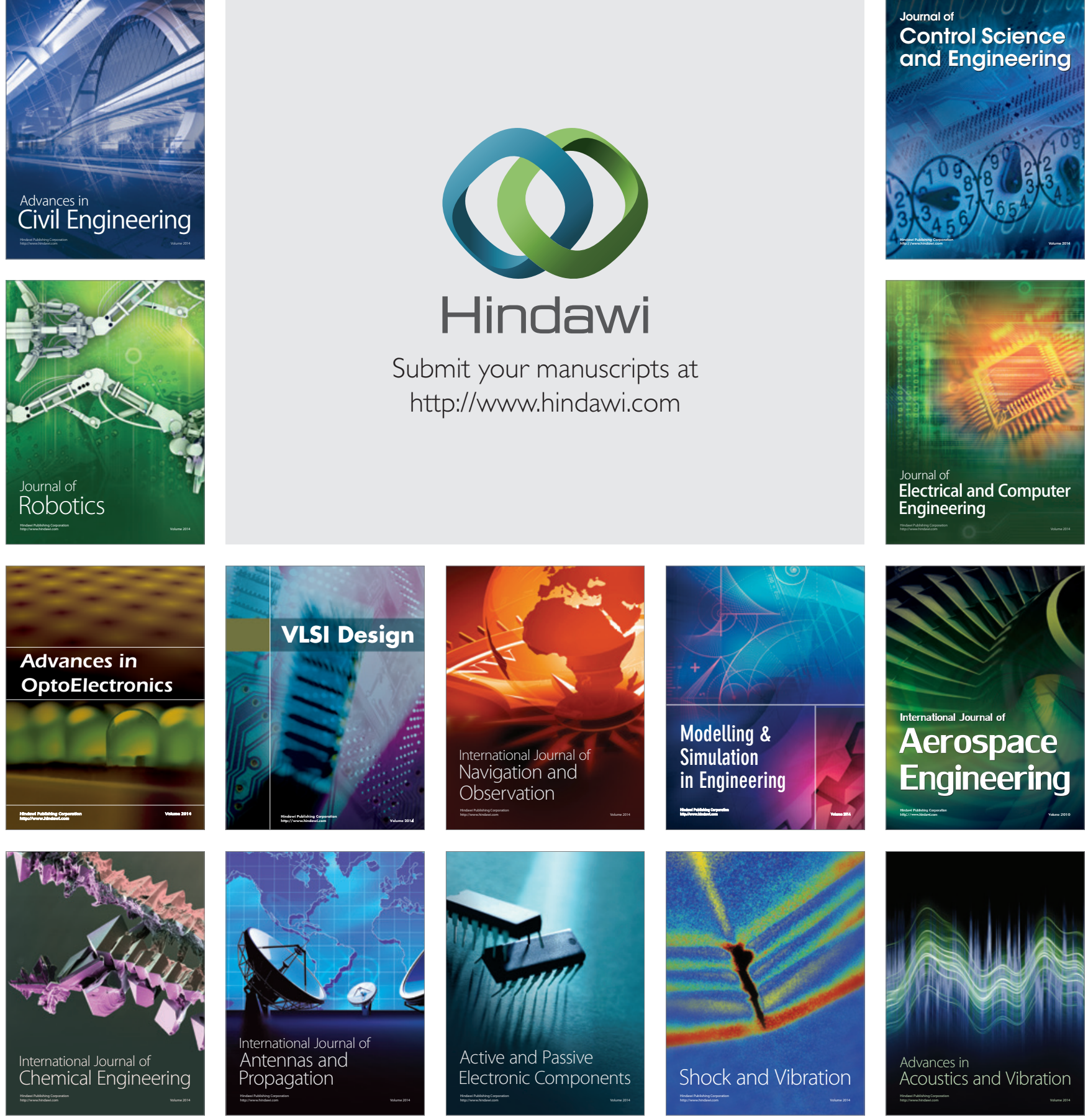